\title{
Visible Light V2V Communication and Ranging System Prototypes Using Spread Spectrum Techniques
}

\author{
Akira John SUZUKI ${ }^{\dagger a)}$, Student Member, Masahiro YAMAMOTO ${ }^{\dagger \mathrm{b})}$, and Kiyoshi MIZUI ${ }^{\dagger \dagger \mathrm{c})}$, Members
}

\begin{abstract}
SUMMARY There is currently much interest in the development of Optic Wireless and Visible Light Communication (VLC) systems in the ITS field. Research in VLC and boomerang systems in particular often remain at a theoretical or computer-simulated level. This paper reports the 3 -stage development of a boomerang prototype communication and ranging system using visible light V2V communication via LEDs and photodiodes, with direct-sequence spread spectrum techniques. The system uses simple and widely available components aiming for a low-cost frugal innovation approach. Results show that while we have to improve the prototype distance measurement unit due to a margin of error, simultaneous communication and ranging is possible with our newly designed prototype. The benefits of further research and development of boomerang technology prototypes are confirmed.
\end{abstract}

key words: ITS, V2V communication, ranging, VLC, spread spectrum

\section{Introduction}

New paradigms in research and development of Intelligent Transport Systems (ITS) are being actively explored to improve and solve road traffic problems such as traffic accidents, congestion, and air pollution [1], [2]. The research field is diverse, with practical application for not only automobiles but also slow or emergency vehicles [3], [4], motorbikes [5] and wheelchairs [6]. In addition, examination of systems such as safe driving support and automatic driving are progressing, along with an understanding of the psychological implications for human drivers and pedestrians [7]-[10]. Integrating technologies that can be used for vehicle-to-vehicle (V2V)/ vehicle-to-infrastructure (V2I) communication in these safe driving or autonomous systems is important. Such hybrid technology enables positioning and distance measurement, providing not only information to your own vehicle, but also offering information and communication with other vehicles, and providing further road information [11]-[14].

In recent years light emitting diode (LED) lighting has rapidly spread as next generation lighting, such as road lighting and signals, vehicle headlights and tail lamps. LEDs are beginning to replace conventional light sources. With this current increase in the regular use of LED lights in

Manuscript received March 14, 2019.

Manuscript revised July 31, 2019.

${ }^{\dagger}$ The author is with Department of Electrical Engineering, Graduate School of Engineering, Kanto Gakuin University, Yokohama-shi, 236-8501 Japan.

${ }^{\dagger}$ The authors are with College of Science and Engineering, Kanto Gakuin University, Yokohama-shi, 236-8501 Japan.

a) E-mail: d15J2001@kanto-gakuin.ac.jp

b) E-mail: yamamoto@kanto-gakuin.ac.jp

c)E-mail: mizui@kanto-gakuin.ac.jp

DOI: 10.1587/transfun.2019TSP0004 vehicles and traffic infrastructure, interest in exploiting the visible light communication (VLC) possibilities and optical wireless communication (OWC) systems for ITS has grown [15], [16]. The benefits of OWC systems over radio frequency include the availability of a license-free frequency spectrum and higher data rates [17]. Since LEDs can blink at a speed imperceptible to human eyes, wireless communication can be executed together with provision of light, frugally optimizing use of technology already featured in vehicles and road infrastructure. However, there are still many challenges to VLC systems, including the need for line-of-sight, possible weather-related signal attenuation, and high-frequency modulation of LEDs without distortion while maintaining comfortable illumination levels. As a result, there is global interest in a rapidly growing body of research into and development of robust and viable systems. Standardizing OWCs such as the Visible Light Communication Consortia (VLCC) and the IEEE standard 802.15.7 [18], [19] has been one area of approach.

Our paper contributes to this global endeavor with an innovative VLC prototype. The paper describes the 3-stage design and testing of our visible light (VL) boomerang prototype using LEDs and photodiodes (PD). Hitherto, Yamazato et al. have researched application of VL to V2I communication using image sensors [20]. The corresponding receptor device for VLC may be either an image sensor or a PD. Although image sensors are highly resistant to interference and capable of using advanced image processing technology, the PDs chosen for our prototype offer lower cost, high-speed communication options.

Mizui et al. have proposed an integrated V2V communication and ranging Boomerang System using spread spectrum techniques which can also be used with LEDs for VLC [21]. The Boomerang System performs V2V communication and ranging simultaneously between two vehicles, with expanded possibilities for communication going back and forth [22]. The Boomerang System has the advantages of simultaneous communication and ranging, its communication media such as radio waves or LED light can be flexibly chosen, has anti-interference characteristics, anti-multipath characteristics, and can hybridize with already existing communication systems.

Although various types of improvements have been proposed for the boomerang system, they have often been studied by computer simulation. One theoretical time-hopping $\mathrm{VL}$ boomerang system has been proposed for $\mathrm{V} 2 \mathrm{~V}$ communication and ranging [23]. This system suggests using an LED 
headlight and a tail lamp mounted on a vehicle. However, this system also remains at the computer simulated level. Kumagai et al. [24] researched an early double boomerang with circuit-delay simulation, and Otsu et al. [25] engaged in experimental demonstration of an infrared and ultrasonic communication-only prototype. We propose closing the gap in VL Boomerang prototype study with our description of the design and research of a VL Boomerang prototype using LEDs and PDs for communication and ranging. In short, this paper offers an original actual prototype as oppose to a theoretical or computer-simulated discussion. The prototype features a novel and distinct VL-based boomerang system. In the boomerang system, previous simulations have been conducted using matched filters for ranging and demodulation. However, in this paper, we propose a new highly usable method, and build an actual prototype with transistortransistor logic (TTL) that can perform demodulation and ranging simultaneously at high speed based on VLC. We suggest a cutting-edge practical and frugal innovation approach in system design using simple and widely available parts.

This paper therefore delineates the 3-stage development of a boomerang prototype communication and ranging system using VL V2V communication via LEDs and PDs, with direct-sequence spread spectrum (DSSS) techniques [26][28]. This 3-stage development comprised: Stage 1 - basic communication system viability check at $5 \mathrm{~cm}$, Stage $2-$ system expanded to $20 \mathrm{~m}$ with use of lenses, and Stage 3 simultaneous distance measurement unit added up to $5 \mathrm{~m}$.

We began with implementation of a basic system viability check with regard to communication. At the communication unit, we applied a simplified approach combining exclusive or (XOR) gate and Low-pass filter. The received signal after amplification, waveform shaping and de-spread was observed in interrogator and responder. Results demonstrated that even subject to diverse interference, VLC was possible. Results also confirmed that ranging may be influenced by prototype processing delay. In the second stage we expanded the system distance viability with an advanced VL boomerang prototype on circuit board, further verifying the feasibility of the initial system for higher speeds and extended distance communication. At the receiving section, light was focused using a lens unit. In experiments, we focused on two scenarios: LED transmitters with and without lens, observing system signals using oscilloscopes. Results confirmed that communication with lens by this second prototype system at a distance of $20 \mathrm{~m}$ was possible. In the third stage we verified the feasibility of performing simultaneous communication and ranging, adding a new distance measurement unit, which is an important feature of the VL boomerang system. At the communication and ranging unit, we also tested our simplified approach combining XOR gate and Low-pass filter. Results confirm that while we have to improve the prototype distance measurement unit due to a margin of error, simultaneous communication and ranging is possible with our newly designed prototype.

This paper is structured as follows: Section 2 explains

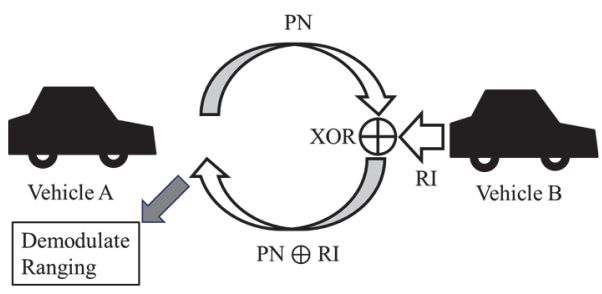

Fig. 1 Basic boomerang system.

the basic boomerang system. In Sect. 3 our prototype VL boomerang system architecture is described. Section 4 highlights our prototype experiment specifications and results, and Sect. 5 discusses the implications of our work and possible future avenues of research and development.

\section{Boomerang System}

Various and improved types of Mizui's boomerang system, a simultaneous $\mathrm{V} 2 \mathrm{~V}$ communication and ranging system using spread spectrum techniques, have been proposed as earlier mentioned.

Figure 1 shows a conceptual diagram of the basic boomerang system. Two vehicles, following vehicle $\mathrm{A}$ and preceding vehicle $\mathrm{B}$, are traveling in proximity. First, vehicle A sends PN, which is a key signal in the spread spectrum techniques, to vehicle $\mathrm{B}$. Next, vehicle $\mathrm{B}$ receives $\mathrm{PN}$, and sends back PN $\oplus$ RI (Responder Information), including vehicle B information such as braking, acceleration, warning signals and change of moving direction for crash prevention. Finally, vehicle A receives this signal from vehicle B, using spread spectrum demodulation XORs the signal by PN (which is the PN code of its own vehicle) to obtain vehicle B information. At this time, the distance between vehicles $\mathrm{AB}$ can be measured from the phase difference corresponding to the signal round-trip time.

\section{Prototype Systems}

In this section, we explain the 3-stage full prototype system created to verify the feasibility of the VL boomerang system in action. Several methods of constructing the circuitry for the VL boomerang method can be considered, but our prototype adopts the DSSS method. The aim was to simplify the implementation of the VLC process with on-off keying (OOK). In addition, using a matched filter may be considered for information demodulation and ranging, but in our prototype machines, a simplified approach using only XOR gate and Low-pass filter (LPF) was adopted. Section 3.1 describes the first and second stage prototypes, and Sect. 3.2 describes the third stage prototype architecture.

\subsection{First and Second Stage Prototypes}

Figure 2 shows the communication unit system diagram of the prototype in the first and second stages. In these two stages, only the communication unit is implemented, with 


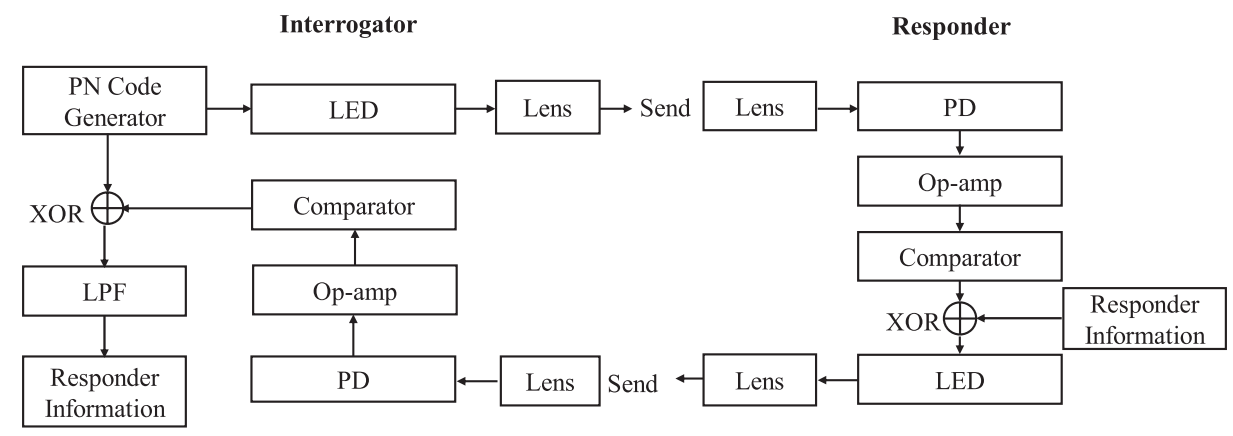

Fig. 2 Communication unit: first and second stage prototype system.

the ranging unit added in the third stage and explained in Sect. 3.2. The difference between the prototypes Stages 1 and 2 is that, in the second stage, lenses are used at LED transmitters and $\mathrm{PD}$ receivers to extend the communication distance. Also, the number of chips is reduced, and the chip rate of the PN code used is increased for higher processing speed. However, the flow of the entire system in both prototypes is basically the same. We therefore describe only the second stage communication unit architecture where lenses are used at the transmitter and receiver.

We describe communication flow in the communication unit as follows: first, the pseudorandom noise (PN) code generated by the interrogator is transmitted to the responder by LED in VLC. The transmitted signal from the interrogator (Interrogator Transmission) is expressed by Eq. (1).

$$
\text { Interrogator Transmission }=P N
$$

At this time, lenses are used to strengthen the transmission: the light is focused by the first lens and received by the PD on the responder side, also through a lens. Then in the responder, the received signal is amplified by the operational amplifier (Op-amp) and compared with the reference voltage by the comparator to make the signal $0 \mathrm{~V}, 5 \mathrm{~V}$. Next, the responder baseband information signal is directly spread by XORing the comparator output. The transmitted signal from the responder (Responder Transmission) is expressed by Eq. (2).

$$
\text { Responder Transmission }=P N^{\prime} \oplus R I
$$

$\mathrm{PN}^{\prime}$ includes both delay due to the one-way distance between interrogator and responder, and processing delay.

It is then sent back via LED in VLC to the interrogator through the lens and captured in the PD. Thereafter, the interrogator Op-amp and comparator refine the signal. Finally, the responder information (RI) is de-spread by XORing the interrogator generated PN code. In this method, phase difference pulses (PDP) as shown in Fig. 3 are generated. Using an LPF to remove PDP, the RI is clearly obtained.

\subsection{Third Stage Prototype}

In the third stage prototype a distance measuring unit is added to the interrogator. Processing in the responder architecture

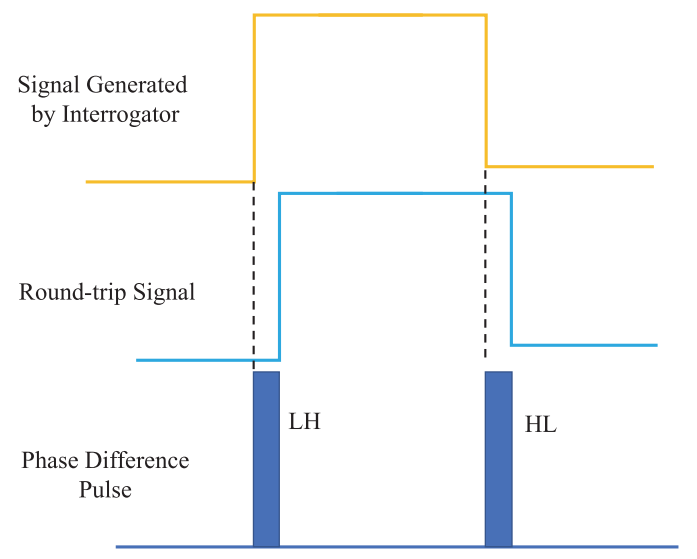

Fig. 3 Phase difference pulses (PDP).

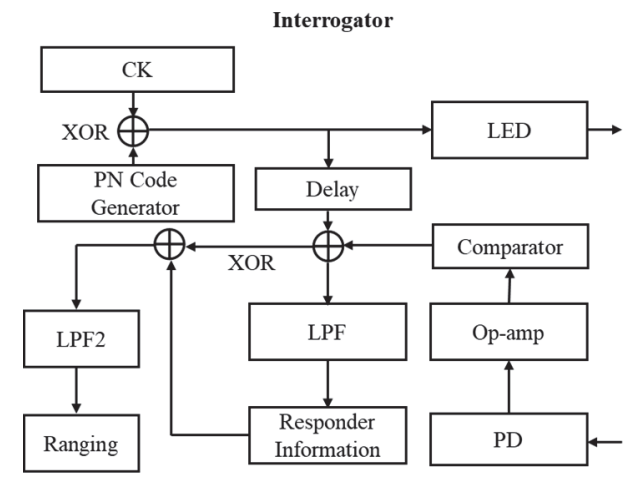

Fig. 4 Third stage interrogator system diagram.

is the same as in the first and second stage prototypes. The system diagram of the interrogator is shown in Fig. 4. First, the interrogator-generated $\mathrm{PN}$ code XORs the reference signal clock pulse (CK) (see Fig. 5). Then in the LED, the electric signal is converted into an optical signal with binary OOK. This optical signal is transmitted to the responder. The transmitted signal from the interrogator is expressed by Eq. (3).

$$
\text { Interrogator Transmission }=C K \oplus P N
$$

During the sending and receiving process, light is focused using a lens and processed through the responder as in the stage 2 prototype: in the responder, the received signal is 


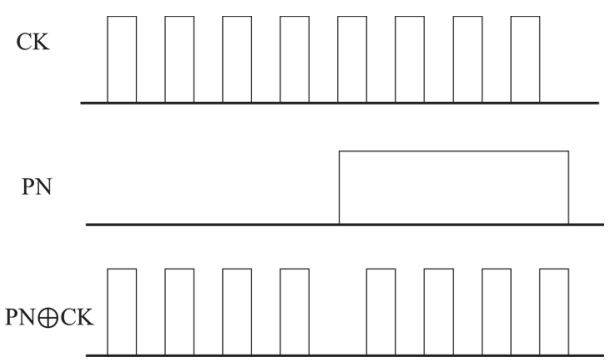

Fig. $5 \quad \mathrm{CK} \oplus \mathrm{PN}$.

amplified by the Op-amp and compared with the reference voltage by the comparator to make the signal $0 \mathrm{~V}, 5 \mathrm{~V}$. Next, the responder baseband information signal is directly spread by XORing the comparator output. The transmitted signal from the responder is expressed by Eq. (4).

$$
\text { Responder Transmission }=(C K \oplus P N)^{\prime} \oplus R I
$$

$(\mathrm{CK} \oplus \mathrm{PN})^{\prime}$ includes both delay due to the one-way distance between interrogator and responder, and processing delay.

Once the interrogator receives the return signal from the responder, it passes through the Op-amp and the comparator. By XORing the $\mathrm{CK} \oplus \mathrm{PN}$ signal of the interrogator, the information of the responder is de-spread. In this method, a PDP corresponding to $\mathrm{CK} \oplus \mathrm{PN}$ is generated. Using an LPF to remove the PDP, the RI is clearly obtained. When performing preliminary experiments at a distance of $0 \mathrm{~m}$, a delay of about $200 \mathrm{~ns}$ had already occurred in the roundtrip signal. This was considered to be processing delay. Therefore, a circuit was added to delay the $\mathrm{CK} \oplus \mathrm{PN}$ signal immediately before XOR.

The distance measuring architecture in this third-stage boomerang prototype is described as follows. By XORing the final RI signal with the de-spread responder signal, PDP itself can be gained. PDP are pulses that vary depending on the phase difference between the transmission signal round trip time, owing to distance and processing delay. By integrating these PDP with the LPF2, we can convert the interrogator-responder round-trip time phase difference as a voltage which the distance measuring unit outputs.

In first and second stage prototypes, only the PN code was transmitted by the interrogator, but in the third stage $\mathrm{CK} \oplus \mathrm{PN}$ is transmitted. The $\mathrm{CK}$ is used to reduce the influence of variation in the number of PDP depending on the $\mathrm{PN}$ code, by controlling the number of PDP at CK frequency. The use of a high frequency CK increases the number of PDP: thus, the output of the distance measuring unit increases greatly according to distance, so that the change in voltage at the distance measuring unit becomes conspicuous.

In addition, as shown in Fig. 3, one PDP appears in the interval immediately after the rising edge (Low-high/LH) and the falling edge (High-low/HL) of the interrogator signal. Since the widths of these two PDP are projected equal, it is possible to extract a voltage corresponding to the distance by integrating these PDP. However, the PDP differed greatly in preliminary experiments in the first and second stage pro- totypes. The pulse width was larger in the HL position than in the LH position. Therefore, in the distance measuring unit, the logical product (AND) with the signal generated by the interrogator is taken to extract only the PDP of LH.

\section{VL Boomerang Prototype Experiments and Results}

In this section, experiment conducted to verify the feasibility of the VL boomerang system are described. The experiment was conducted indoors (Kanto Gakuin University Building 6, 6th floor; $\left.35^{\circ} 19^{\prime} 19.2^{\prime \prime} \mathrm{N} 139^{\circ} 37^{\prime} 23.5^{\prime \prime} \mathrm{E}\right)$. The prototype specifications are shown in Table 1. The main ICs and manufacturers used in the prototypes are shown in Table 2.

\subsection{First Stage Prototype Experiments}

\subsubsection{First Stage Prototype Specifications}

The aim of this initial prototype was to build and confirm the viability of an LED VL boomerang communication system in action. From Table 1, the PN code chip rate is $10 \mathrm{kchip} / \mathrm{s}$. An m-sequence of 127 chips was used for PN code. The transmission method was OOK without lenses. LEDs used for both interrogator and responder were red. The information bit rate was $79 \mathrm{bits} / \mathrm{s}$, and the communication distance was $5 \mathrm{~cm}$. The signals of interrogator and responder were first observed with an oscilloscope in order to confirm the essential performance and viable operation of the system design. We also conducted basic interference experiments with both interrogator and responder PDs. Various kinds of interference waves are conceivable, but in this experiment, a different $\mathrm{m}$-sequence of 127 chips to the one used for communication was employed. In the experiment the LEDs and the PDs of the interrogator and the responder were line-ofsight aligned. Interference LEDs at half the luminance were set up next to communication LEDs to check interference at each PD in turn.

\subsubsection{First Stage Prototype Results}

Firstly, we confirmed that the prototype does operate as projected under ideal conditions, i.e. it is communicating well for the specified $5 \mathrm{~cm}$. These initial confirmations were gained by observing the waveform on the oscilloscope. Subsequently interference experiments established a challenging pattern of disturbance: the oscilloscope waveforms documented that the signal after responder amplification was indeed disturbed due to the influence of the interference LED. Also exiting the comparator, signal disturbance was identified. Due to this negative influence, errors occurred in the signal received by the interrogator. The communication is disturbed by the interference, but it was confirmed that the original RI signal could nevertheless be demodulated to some extent and communication was maintained. 
Table 1 Prototype specifications.

\begin{tabular}{|c|c|c|c|}
\hline Category & First Stage & Second Stage & Third Stage \\
\hline CK & none & none & $1 \mathrm{MHz}$ \\
\hline PN Chip Rate & $10 \mathrm{kchip} / \mathrm{s}$ & 1 Mchip/s & $250 \mathrm{kchip} / \mathrm{s}$ \\
\hline Bit Rate & $79 \mathrm{bit} / \mathrm{s}$ & $10 \mathrm{kbps}$ & $10 \mathrm{kbps}$ \\
\hline PN Code & $127 \mathrm{chip}$ & $15 \mathrm{chip}$ & $15 \mathrm{chip}$ \\
\hline Modulation & OOK & OOK & OOK \\
\hline Distance & $5 \mathrm{~cm}$ & $20 \mathrm{~m}$ & $5 \mathrm{~m}$ \\
\hline Use of Lens & without & with & with \\
\hline Interrogator & Red, & White, & White, \\
LED & $75 \mathrm{~cd}, 15^{\circ}$ & $75 \mathrm{~cd}, 15^{\circ}$ & $75 \mathrm{~cd}, 15^{\circ}$ \\
\hline Responder LED & $\begin{array}{c}\text { Red, } \\
75 \mathrm{~cd}, 15^{\circ}\end{array}$ & $\begin{array}{c}\text { Red, } \\
75 \mathrm{~cd}, 15^{\circ}\end{array}$ & $\begin{array}{c}\text { Red, } \\
75 \mathrm{~cd}, 15^{\circ}\end{array}$ \\
\hline
\end{tabular}

Table 2 ICs and manufacturers.

\begin{tabular}{|c|c|c|c|}
\hline Category & First Stage & Second Stage & Third Stage \\
\hline \multirow{2}{*}{$\begin{array}{c}\text { PN Code } \\
\text { Generator }\end{array}$} & $\begin{array}{c}\text { PIC16F648A } \\
\text { Microchip }\end{array}$ & $\begin{array}{r}\text { SN74HC164N, SN74HC86 } \\
\text { Texas Instruments }\end{array}$ \\
\hline \multirow{2}{*}{ Op-amp } & $\begin{array}{c}\text { NJM072D } \\
\text { NJR }\end{array}$ & $\begin{array}{c}\text { LMH6702MA } \\
\text { National Semiconductor }\end{array}$ \\
\hline \multirow{2}{*}{ Comparator } & NJM072D & NJM360D \\
\cline { 2 - 3 } & \multicolumn{2}{|c|}{ New Japan Radio (NJR) } \\
\hline \multirow{2}{*}{ PD } & S2506-02 & S6967 \\
\cline { 2 - 3 } & \multicolumn{3}{|c|}{ Hamamatsu Photonics K.K. } \\
\hline
\end{tabular}

\subsection{Second Stage Prototype Experiments}

\subsubsection{Second Stage Prototype Specifications}

In the second stage prototype we increased communication speed, changing the low speed IC and PD from prototype 1 to a high-speed IC and PD as in Table 1. Also, we increased communication distance to $20 \mathrm{~m}$ using lenses. The interrogator and the responder together with their power supply were placed on two $0.8 \mathrm{~m}$ high motionless tables. LED colors were switched to white and red respectively since boomerang VLC is assumed between headlight and tail lamp. The PN code chip rate was increased 100-fold to $1 \mathrm{Mchip} / \mathrm{s}$ and the information bit rate is $10 \mathrm{kbps}$. An m-sequence of 15 chips was used for PN code. In practice, a longer $\mathrm{m}$-sequence as in prototype 1 may be preferable, but in this experiment a short $\mathrm{m}$-sequence was easier to observe with the oscilloscopes. Due to the long distance between interrogator and responder, the waveform was observed using two oscilloscopes. Two $6 \mathrm{~cm}$ diameter lenses were placed together to form one lens for focal length. First, we conducted experiments using lenses at each transmitter and receiver of interrogator and responder as shown in Fig. 6. The experimental distance in this case was $0-20 \mathrm{~m}$ between the lenses. This experiment assumed stronger light comparable to a vehicle

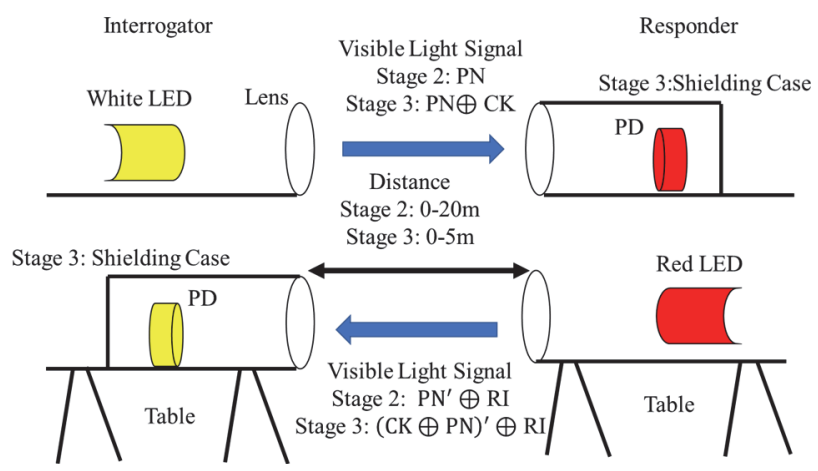

Fig. 6 Second and third stage prototypes experiments.

headlight. Illuminance of the white LED was approximately $10001 \mathrm{x} / 1000 \mathrm{~cd}$ after passing the lens, measured at a distance of $1 \mathrm{~m}$ with an LX-1010 B illuminometer. This is, however, still darker than a headlight. Next, we conducted a second experiment assuming weak light such as distant vehicles and traffic lights. For this purpose, the transmitter of the interrogator and responder used no lenses. The experimental distance was set at $50 \mathrm{~cm}$ intervals up to $2.5 \mathrm{~m}$ between the LED and the lens. In both experiments the LEDs and the PDs of the interrogator and the responder were line-of-sight aligned.

\subsubsection{Second Stage Prototype Results}

Our first experiment (using lenses at transmitter and receiver of both interrogator and responder) confirmed good performance. Longer distance boomerang VL communication was viable up to $20 \mathrm{~m}$. Figure 7(a) (1)-(4) and 7(b) (1)-(8) shows resulting waveforms observed in interrogator and responder oscilloscopes respectively for a distance of $20 \mathrm{~m}$. Interrogator and responder signal graphs are not perfectly time synchronized due to the accuracy of human performance. Waveform observed at signal observation points (1)-8) are as follows:

(1) At interrogator LED

(2) At amplified output of interrogator PD

(3) After passing through interrogator comparator

(4) At demodulated RI signal

(5) At amplified output of transponder PD

(6) After passing through responder comparator

(7) At responder LED

(8) Before sending responder-generated information

Oscilloscope observation results (4) and (8) confirmed that the RI was correctly demodulated in the interrogator at $20 \mathrm{~m}$. Communication at a longer distance than $20 \mathrm{~m}$ was no longer viable.

Our second experiment (without lenses for the transmission section of interrogator and responder) measured weaker light conditions. However, even in this case, we confirmed that the prototype was communicating well. The RI was correctly demodulated up to but not beyond $2.5 \mathrm{~m}$. Overall these results confirm a viable long-distance VL communica- 
(1)

(2)

(3)

(4)

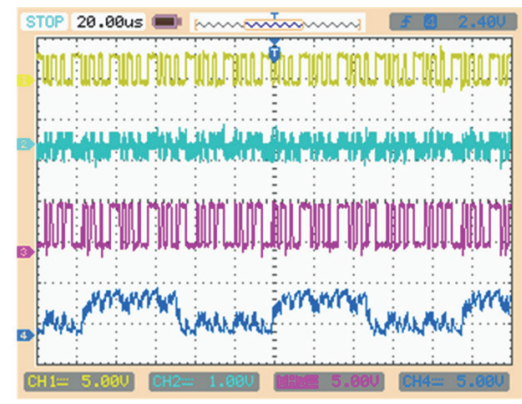

(a) Interrogator signals.

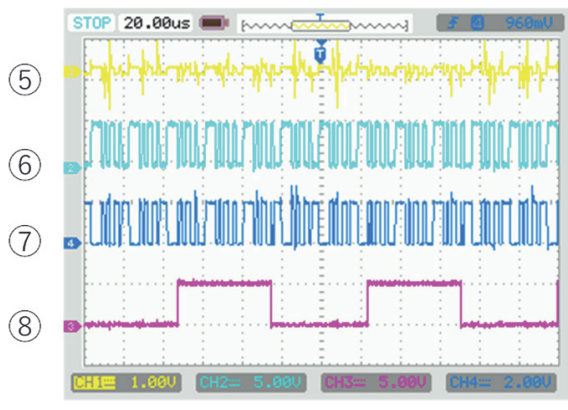

(b) Responder signals.

Fig. 7 Second stage experiment waveforms observed in oscilloscopes for a distance of $20 \mathrm{~m}$ (with lenses).

tion capability for our boomerang prototype.

\subsection{Third Stage Prototype Experiments}

\subsubsection{Third Stage Prototype Specifications}

Having established the success of the high-speed second prototype, our aim with the third-stage prototype was to incorporate and test the distance measuring unit of the $\mathrm{VL}$ boomerang system design. In order to maintain the highspeed nature of the design, the CK frequency used in the experiment was $1 \mathrm{MHz}$, the $\mathrm{PN}$ code chip rate was $250 \mathrm{kchip} / \mathrm{s}$, and the RI bit rate was $10 \mathrm{kbps}$. The experimented distance was $0-5 \mathrm{~m}$. Both lenses and LEDs were the same specifications as in the second stage prototype (See Table 1). A diagram of the experiment is shown in Fig. 6. The lenses and the PD were covered with a light shielding case to shield from background light. Oscilloscope waveforms were observed by moving each LED at approximately $50 \mathrm{~cm}$ intervals with the distance of $0-5 \mathrm{~m}$ in line-of-sight alignment.

The two types of voltage output, LH alone and both LH and HL together, were measured at each point five times with a multimeter to confirm the precision of the distance measuring unit. The pulse width was larger in the HL position than in the LH position. Therefore, in the distance measuring unit, the logical gate (AND) was used to extract only the LH PDP.

\subsubsection{Third Stage Prototype Results}

First, we explain the waveforms observed with the oscillo-

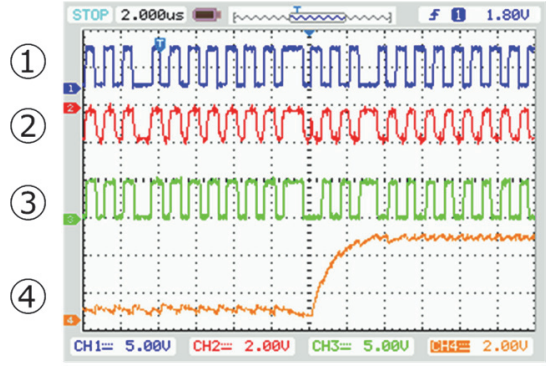

(a) Interrogator signals.

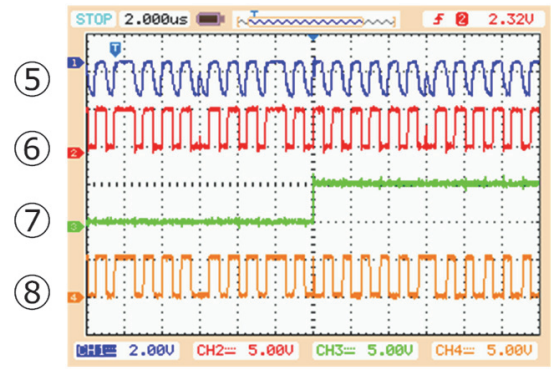

(b) Responder signals.

Fig. 8 Third stage experiment waveforms observed in oscilloscopes for a distance of $0 \mathrm{~m}$.

scopes. Figure 8(a) (1)-(4) and 8(b) (5)-(8) shows resulting waveforms observed in interrogator and responder oscilloscopes respectively for a distance of $0 \mathrm{~m}$. Waveform observed at signal observation points (1)-8) are as follows:

(1) At interrogator-generated $\mathrm{CK} \oplus \mathrm{PN}$

(2) At amplified output of interrogator PD

(3) After passing through interrogator comparator

(4) At demodulated RI signal

(5) At amplified output of transponder PD

(6) After passing through responder comparator

(7) Before sending responder-generated information

(8) Before entering responder LED

Figure 8 results confirm that the prototype is functioning as designed, i.e. the prototype was communicating well. In (1) and (2) a part of the signal is inverted due to the RI, which is as expected. The clean lines in (3) show the comparator is functioning accurately. A comparison of (4) and (7) shows the baseband signal transmitted by the interrogator has been demodulated, verifying that the interrogator is operating correctly. Likewise, results from (5) (6) (7) in Fig. 8(b) confirm that the responder is also operating correctly. From these observations, we conclude that communication between the interrogator and the responder is occurring as designed. Although the demodulated signal became increasingly unstable as the distance was increased, similar waveforms were obtained up to $5 \mathrm{~m}$. After $5.5 \mathrm{~m}$, demodulating RI was no longer possible in this prototype design.

Second, we explain the distance measuring unit results. Figure 9 shows a distance measuring unit output graph of the actual voltage (Output1) together with a projected value (Equation) obtained by measuring the PDP of LH alone. The projected value in the line graph was determined by the 


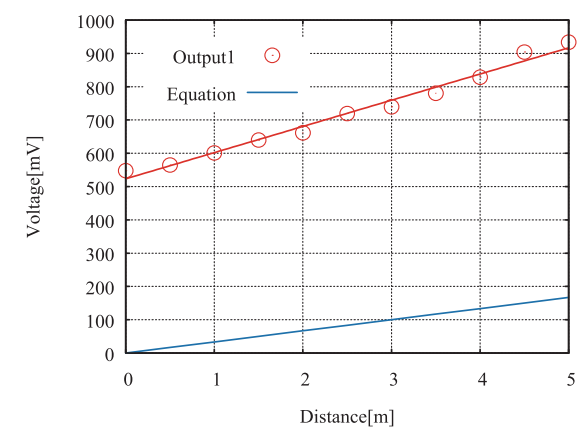

Fig.9 The result of measuring unit PDP voltage of LH alone.

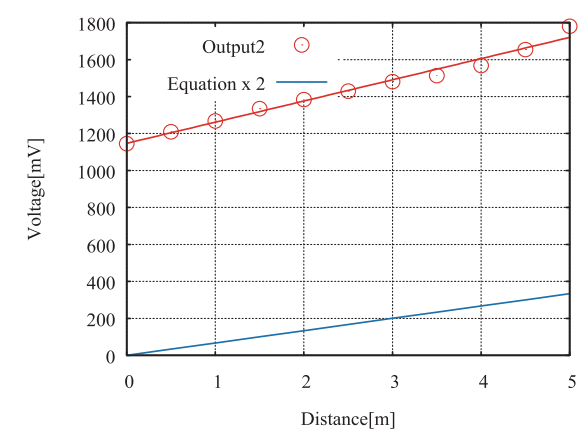

Fig. 10 The result of measuring unit PDP voltage of both LH and HL.

Eq. (5).

$$
\text { Vout }=\frac{2 D f V}{c}
$$

c: speed of light, D: distance to target, $f$ : clock frequency, $\mathrm{V}$ : voltage of output ' 1 '. However, this projection was conceived only as a guideline: the equation does not take into account the PN code edge and processing delay among others. Although we added a circuit as explained in Sect. 3.2 to alleviate the processing delay in advance, results diverged greatly from our projected value. Moreover, as seen, the gradient is larger than projected, i.e. in this particular prototype the ranging performance is low. Divergence between output 1 and projected value needs to be resolved to facilitate precision measuring.

Next, Fig. 10 shows the result of measuring the PDP of both LH and HL together (Output2) and the projected value (Equation $\times 2$ ). There are two pulses, so the projected value is doubled. Again, both projected values and gradients are divergent. One reason is that the processing delay increases with the amount of light. When the light intensity on the PD was varied for equal distance, a change was observed in the output voltage. More intensity resulted in lower output, while less intensity resulted in higher output. Since the amount of light entering the PD inevitably decreases as distance increases, the gradient is larger than the projected value.

The second reason is the change in duty cycle due to the round-trip signal. When comparing the interrogator LED transmission signal and PD received signal in the oscilloscope, the duty cycle was observed to be different. As the duty cycle changes, the output voltage of the distance measuring unit increases and decreases accordingly, leading to a divergence between output and our projected value.

The third reason is the upward sloping rise in the waveform observed at demodulated RI signal (4). The rise time is slow. This delay is caused at the LPF used for demodulation. This distorts the PDP. However, this error can be minimized by increasing the clock frequency and decreasing the bit rate.

While these three issues must be addressed in order to minimize the divergence between output voltage and projected values, we consider that ranging will work well if we process the output appropriately.

Overall results presented in this section confirm our VL boomerang system prototype had good communication performance and show the feasibility of conducting simultaneous communication and ranging in our design.

\section{Conclusion}

In this paper, we report the creation of the first actual prototype of a VL boomerang system. The design was advanced in three stages as viability and reliability were tested and researched.

First stage: Prototype designed for checking smooth communication of VL boomerang system. Results confirmed that communication by our VL boomerang system is possible even if interference is mixed to some extent.

Second stage: Prototype with improved response speed and extended maximum communication distance using lenses. Results verified VL communication at a practical distance of $20 \mathrm{~m}$.

Third stage: Prototype designed with lenses and communication unit, adding a distance measurement unit. Results confirm a maximum communication and ranging distance of $5 \mathrm{~m}$.

The aim of this research was to explore the possibilities of a practical and frugal VLC system which uses already existing features on automobiles. The prototype design explored benefits of using low cost devices such as PDs, LEDs, LPF and XOR gate with a global mass market approach in mind. The VLC system is ideal for combining with other onboard systems such as GPS and laser for rich data input and added reliability and safety. Our prototype experiments confirm further high-speed VLC potential with this LED PD combination.

Since the basic reliability and feasibility of this VLC approach has now been demonstrated, further research to develop on-board prototypes for mounting in vehicles is suggested. The LEDs in the prototype performed with lower luminescence than vehicle headlights. Actual headlights may offer an even further communication distance potential. Researching the issue of how vehicle vibration and natural light including adverse weather may impact the system is also relevant.

Challenges remain. The calibration of the ranging device, including fine-tuning the issue of divergence must be addressed. Due to CK influence the third prototype system 
validity dropped to a distance of $5 \mathrm{~m}$, but $\mathrm{CK}$ was beneficial for ranging. This issue must also be researched. Moreover, since the system uses lenses, the focus in the PD may be more susceptible to line-of-sight alignment issues. However, this feature has potential to be exploited positively to avoid unwanted interference in the communication.

Overall, we suggest the development of this VL communication prototype is an exciting step forward in the "new paradigm of road traffic" [1].

\section{Acknowledgments}

We are grateful to Prof. T. Hasegawa for the University of Saitama for helpful discussions and valuable comments. This work was supported by JSPS KAKENHI Grant Number $15 \mathrm{~K} 01234$.

\section{References}

[1] T. Nagaosa, "FOREWORD," IEICE Trans. Fundamentals, vol.E100A, no.2, p.431, Feb. 2017.

[2] MLIT Homepage, http://www.mlit.go.jp/en/index.html, Retieved March 2019.

[3] T. Manabe and T. Hasegawa, "On SV probe data collection and analysis platform," IEICE Technical Report, vol.116, no.70, pp.1318, May 2016.

[4] H. Nanba, Y. Ikami, K. Imai, K. Kobayashi, and M. Sawada, "An advantage of the vehicle to vehicle communication for an automated driving car at the encounter with an ambulance," IEICE Trans. Fundamentals, vol.E101-A, no.9, pp.1281-1289, Sept. 2018.

[5] S. Kimura, H. Hiroyuki, M. Fujii, A. Ito, Y. Watanabe, and T. Kitani, "Behavior estimation method based on movement trajectory by the position information," IEICE Trans. Fundamentals, vol.E101-A, no.9, pp.1317-1319, Sept. 2018.

[6] T. Sawabe, M. Kanbara, and N. Hagita, "Comfortable intelligence for evaluating passenger characteristics in autonomous wheelchairs," IEICE Trans. Fundamentals, vol.E101-A, no.9, pp.1308-1316, Sept. 2018.

[7] H. Tanaka, AA. Kotani, K. Nishi, and K. Oguri, "A study of the sense of security needed for the input devices of the vehicle in the vicinity of the time to collision," IEICE Trans. Fundamentals, vol.E100-A, no.2, pp.473-481, Feb. 2017

[8] T. Wada, G. Nakagami, and S. Kawai, "New estimation method of pedestrian's running out into road by using pressure sensor and moving record for traffic safety," IEICE Trans. Fundamentals, vol.E100A, no.2, pp.482-490, Feb. 2017.

[9] O. Derbel and R. Landry, Jr., "Driver behavior assessment in case of critical driving situations," IEICE Trans. Fundamentals, vol.E100-A, no.2, pp.491-498, Feb. 2017.

[10] H. Tanaka, D. Takemori, T. Miyachi, Y. Iribe, and K. Oguri, "Analysis of driver's anxiety and security during the braking of a vehicle," IEICE Trans. Fundamentals, vol.E100-A, no.2, pp.466-472, Feb. 2017.

[11] Y. Takatori and H. Takeo, "Analysis of vehicle information sharing performance of an intersection collision warning system," IEICE Trans. Fundamentals, vol.E100-A, no.2, pp.457-465, Feb. 2017.

[12] A.U. Peker and T. Acarman, "VANET-assisted cooperative vehicle mutual positioning: Feasibility study," IEICE Trans. Fundamentals, vol.E100-A, no.2, pp.448-456, Feb. 2017.

[13] T. Acarman and C. Göçmenoğlu, "Texture-based satellite visibility detection for efficient 3D-model-aided GNSS," IEICE Trans. Fundamentals, vol.E100-A, no.2, pp.432-439, Feb. 2017.

[14] J.E. Siegel, D.C. Erb, and S.E. Sarma, "A survey of the connected vehicle landscape - Architectures, enabling tchnologies, applications, and development areas," IEEE Trans. Intell. Transp. Syst., vol.19, no.8, pp.2391-2406, Aug. 2018.

[15] M. Nakagawa (Supervise), VLCC (Editor), "Kashikou Tsuushin no Sekai - LED de Hiraku Akari Komyunike-syon," (The world of visible optical communication - It opens with LED - "Light Communications"), Kougyou Chosakai, Jan. 2006 (in Japanese).

[16] Y. Zhuang, L. Hua, L. Qi, J. Yang, P. Cao, Y. Cao, Y. Wu, J. Thompson, and H. Haas, "A survey of positioning systems using visible LED lights," IEEE Commun. Surveys Tuts., vol.20, no.3, pp.1963-1988, Third Quarter 2018.

[17] S. Arbibm, M. Uysal, Z. Ghassemlooy, Z. Xu, and J. Cheng, "Guest editorial: Optical wireless communications," IEEE J. Sel. Areas Commun., vol.33, no.9, pp.1733-1737, Aug. 2015.

[18] VLCC Homepage, http://www.vlcc.net/?ml_lang=en, Retrieved March 2019.

[19] IEEE802.15.7, http://www.ieee802.org/15/pub/TG7.html, Retrieved March 2019.

[20] T. Yamazato, I. Takai, H. Okada, T. Fujii, T. Yendo, S. Arai, M. Andoh, T. Harada, K. Yasutomi, K. Kagawa, and S. Kawahito, "Imagesensor-based visible light communication for automotive applications," IEEE Commun. Mag., vol.52, no.7, pp.88-97, 2014.

[21] K. Mizui, M. Uchida, and M. Nakagawa, "Vehicle-to-vehicle communication and ranging system using spread spectrum technique," IEICE Trans. Commun. (Japanese Edition), vol.J78-B-2, no.5, pp.342-349, May 1995.

[22] A.J. Suzuki and K. Mizui, "Bidirectional vehicle-to-vehicle communication and ranging systems with spread spectrum techniques using laser radar and visible light," IEICE Trans. Fundamentals, vol.E100A, no.5, pp.1206-1214, May 2017.

[23] K. Mizui, "A study on vehicle-to-vehicle communication and ranging system using spread spectrum technique with visible light," IEICE Technical Report, ITS2012-69, 2013 (in Japanese).

[24] S. Kumagai, J. Kurihara, K. Wada, and K. Mizui, "A prototype of 2-way communication and ranging system using spread spectrum technique," IEICE Technical Report, 100(649), pp.37-42, March 2001 (in Japanese).

[25] Y. Ohtsu and K. Mizui, "A study on vehicle-to-vehicle communication demonstration system using communication part of SS boomerang transmission system," IEICE Technical Report, ITS 105(104), pp.17-21, May 2005 (in Japanese).

[26] A.J. Suzuki, M. Yamamoto, and K. Mizui, "A prototype visible light $\mathrm{V} 2 \mathrm{~V}$ communication and ranging system using spread spectrum technique,” ITS 2016(1-20), pp.35-39, March 2016 (in Japanese).

[27] A.J. Suzuki, K. Futaki, M. Yamamoto, and K. Mizui, "A prototype visible light $\mathrm{V} 2 \mathrm{~V}$ communication and ranging system using spread spectrum techniques (2nd Report)," IEICE Technical Report, 117(102), pp.31-36, June 2017 (in Japanese).

[28] A.J. Suzuki, M. Yamamoto, and K. Mizui, "A prototype visible light V2V communication and ranging system using spread spectrum technique (3rd Report)," IEICE Technical Report, vol.118, pp.49-54, Feb. 2019 (in Japanese).

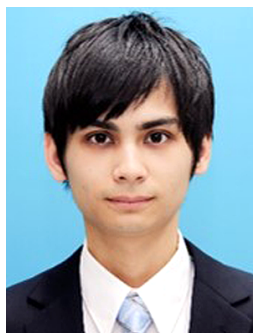

Akira John Suzuki received B.S. and M.S degrees in Electrical Engineering from Kanto Gakuin University in 2013 and 2015 respectively. Currently Ph.D. student at Kanto Gakuin University, his main research interest includes Intelligent Transport Systems and V2V communications. 


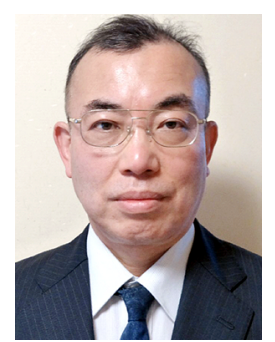

Masahiro Yamamoto received B.E., M.E., and Dr. Eng. degrees from Keio University in 1978, 1980, and 1983, respectively. Since 1985, he has been with Kanto Gakuin University and is now a professor of College of Science and Engineering. His current research area is circuits of communication systems. He is a member of IEICE.

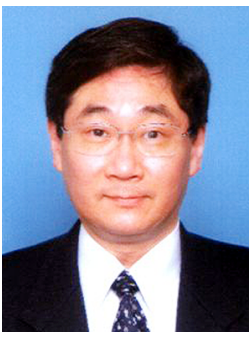

Kiyoshi Mizui received B.E., M.E., and Dr. Eng. degrees from Keio University in 1985, 1987, and 1990, respectively. Since 1990, he has been with Kanto Gakuin University and is now a professor of College of Science and Engineering. His current research area is communication systems and ranging systems of Intelligent Transport Systems (ITS). He is a member of IEICE, Society of Automotive Engineers of Japan, The Japan Society of Applied Science, and IEEE. 